University of Nebraska - Lincoln

DigitalCommons@University of Nebraska - Lincoln

May 2008

\title{
Optimization of Magneto-Optical Kerr Setup: Analyzing experimental assemblies using Jones matrix formalism
}

Christian Binek

University of Nebraska, Lincoln, cbinek@unl.edu

Follow this and additional works at: https://digitalcommons.unl.edu/physicsbinek

Part of the Physics Commons

Binek, Christian, "Optimization of Magneto-Optical Kerr Setup: Analyzing experimental assemblies using Jones matrix formalism" (2008). Christian Binek Publications. 60.

https://digitalcommons.unl.edu/physicsbinek/60

This Article is brought to you for free and open access by the Research Papers in Physics and Astronomy at DigitalCommons@University of Nebraska - Lincoln. It has been accepted for inclusion in Christian Binek Publications by an authorized administrator of DigitalCommons@University of Nebraska - Lincoln. 


\title{
Optimization of magneto-optical Kerr setup: Analyzing experimental assemblies using Jones matrix formalism
}

\author{
S. Polisetty, J. Scheffler, S. Sahoo, Yi Wang, T. Mukherjee, Xi He, and Ch. Binek ${ }^{\text {a) }}$ \\ Department of Physics and Astronomy and the Nebraska Center for Materials and Nanoscience, \\ University of Nebraska-Lincoln, Nebraska 68588-0111, USA
}

(Received 17 December 2007; accepted 1 May 2008; published online 28 May 2008)

\begin{abstract}
We present a comparative study on an experimental and theoretical optimization of magneto-optical Kerr setups based on photoelastic modulation and phase sensitive detector methodology. The first and second harmonics, $I_{\omega, 2 \omega}$, of the reflected light intensity are measured for a $\mathrm{CoO} / \mathrm{Co}$ magnetic reference film. The magnetic field dependence of the optical off-diagonal Fresnel reflection coefficients $r_{p s}$ and $r_{s p}$ follows the sample magnetization. Different Kerr setups provide various dependencies of $I_{\omega, 2 \omega}$ on the reflection coefficients and, hence, on the Kerr ellipticity $\varepsilon_{K}$ and rotation $\theta_{K}$. Jones matrix formalism has been used to analyze the impact of a systematic variation of relative analyzer and polarizer orientations with respect to each other and with respect to the retardation axis of the modulator involved in longitudinal Kerr setups for incoming s-polarized light. We find one particular setup which maximizes $I_{\omega}$ as well as $I_{2 \omega}$ and maximizes the signal-to-noise ratio. Inefficient setups are characterized by $I_{\omega, 2 \omega}$ intensities involving large nonmagnetic contributions of $r_{p}$ and $r_{s}$. (C) 2008 American Institute of Physics. [DOI: 10.1063/1.2932445]
\end{abstract}

\section{INTRODUCTION}

The magneto-optical Kerr effect ${ }^{1}$ (MOKE) has emerged as a powerful experimental technique to study the magnetic properties of thin films and multilayers. Compared to other elegant techniques such as superconducting quantum interference device magnetometry, magnetic force microscopy, etc., the advantages of MOKE include high sensitivity down to the monolayer resolution, ${ }^{2,3}$ high temporal and spatial resolution, simplicity, and straightforward in situ implementation all of that at very low costs in comparison with its alternatives. MOKE has been extensively employed to investigate several important phenomena of modern magnetism. Some of them are: determination of electronic structure, ${ }^{4}$ discovery of the oscillations in exchange coupling between ferromagnetic layers separated by an antiferromagnetic (or nonmagnetic) layer, ${ }^{5-7}$ observation of perpendicular anisotropy in ultrathin films, ${ }^{8}$ test of two-dimensional Ising model in monolayer films, ${ }^{9}$ spin reorientation transition, ${ }^{10-13}$ and correlation between magnetic anisotropy and lattice symmetry breaking. ${ }^{14}$

MOKE measurements can be realized with various setups. Here we focus on the analysis of the widely used modulation technique. Following the path of the light beam from its starting place to the photodetector, the setup involves a monochromatic light source which can be realized, e.g., by a stable laser diode. The latter provides a monochromatic, nearly parallel light beam of roughly linearly polarized light. Further elements are a polarizer $(\mathrm{P})$, the magnetic sample $(\mathrm{S})$, a photoelastic modulator $(\mathrm{O})$, an analyzer $(\mathrm{A})$, and the photodetector. Since the Kerr rotation angle $\theta_{K}$ and ellipticity $\varepsilon_{K}$ are typically small, i.e., $\sim 10^{-3} \mathrm{rad}$, optimization of the

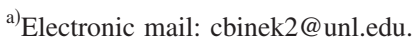

signal-to-noise $(\mathrm{S} / \mathrm{N})$ ratio is very crucial. In order to achieve an optimum $\mathrm{S} / \mathrm{N}$ ratio, appropriate placements and orientations of the optical components are crucial. By now, a great variety of MOKE methodologies, both experimentally and analytically, have been developed. ${ }^{15-25}$ However, a systematic investigation of the $\mathrm{S} / \mathrm{N}$ ratio, both experimentally and theoretically, for all possible configurations of optical elements is still lacking.

In this paper, we analyze a large variety of commonly used arrangements of the optical elements where the position of $\mathrm{O}$ and the relative orientations of $\mathrm{P}$ and $\mathrm{A}$ with respect to each other and with respect to modulator retardation axis have been systematically changed. We use a modulation technique allowing the application of phase sensitive detection methodology by means of a lock-in amplifier. Although the modulation technique is in principle widely employed, ${ }^{26-31}$ there can be various arrangements of the optical components which yield similar but not identical results from the point of view of $\mathrm{S} / \mathrm{N}$ optimization. In particular, experiments studying the evolution of magnetic properties involving the magnetic history of subsequently cycled loops, e.g., aging phenomena such as the training of the exchange bias effect, ${ }^{32}$ cannot just average loops to increase the $\mathrm{S} / \mathrm{N}$. Therefore, an $\mathrm{S} / \mathrm{N}$-optimized configuration is mandatory. Moreover, it is often assumed that the Kerr ellipticity as well as the Kerr rotation are directly proportional to the magnetization; however, studies in multilayer structures reveal that more careful analysis can be in order and both Kerr rotation and ellipticity should be analyzed and compared. ${ }^{33}$

By using Jones matrix formalisms, we identify an optimized Kerr configuration and confirm its superior performance experimentally. We find that the optimum setup is somewhat counterintuitive. In the latter only partial modulation of the polarization of the Kerr rotated signal takes place 


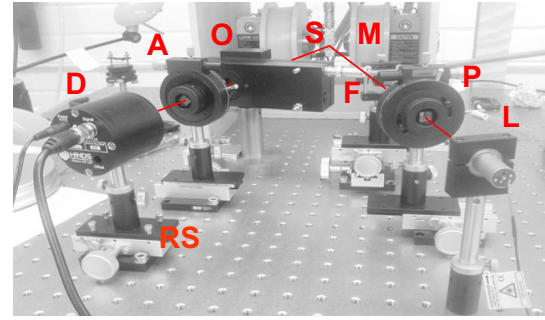

(a)

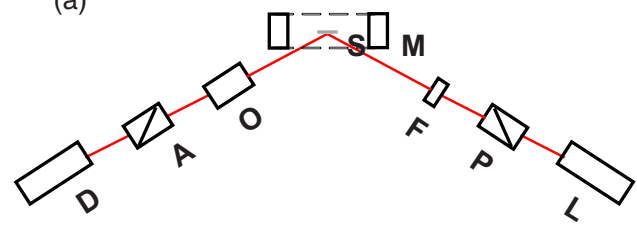

(b)

FIG. 1. (Color online) (a) A photograph of the MOKE measurement setup in longitudinal geometry. L: laser, P: polarizer, F: focusing lens, M: magnet pole, S: sample, O: elasto-optic modulator, A: analyzer, D: detector. P, F, A, and $\mathrm{D}$ are mounted on rotating stages $R S$. (b) A schematic of the longitudinal MOKE.

in contrast to a complete modulation between left and right circularly polarized light. We also emphasize the fact that when simultaneously measuring the first and second harmonics this appropriate optimized configuration discussed in detail below should be used. Our theoretical conclusions are experimentally confirmed by measuring hysteresis loops on a sample $c$-plane $\mathrm{Al}_{2} \mathrm{O}_{3} / \mathrm{Co}[10 \mathrm{~nm}$ hcp (0002)]/CoO $(2.5 \mathrm{~nm}$ naturally ex situ formed polycrystalline oxide). Corresponding $\mathrm{S} / \mathrm{N}$ ratios for each configuration are calculated. Although we present those results involving only the case of longitudinal MOKE which utilizes $s$-polarized light; the analyses can easily be extended to the cases of polar and transverse MOKE as well as for $p$-polarization state.

\section{EXPERIMENTAL SETUP}

Figure 1(a) shows a photograph of our MOKE measurement setup while the corresponding schematic drawing is shown in Fig. 1(b). It starts with a solid state laser diode of wavelength $\lambda=670 \mathrm{~nm}$ and an output power of $5 \mathrm{~mW}$. The latter produces a nearly linearly polarized beam allowing for $s$-polarized (electric field vector oscillating perpendicular to the plane of incidence) or $p$-polarized (electric field vector oscillating in the plane of incidence) configurations. Subsequently, we discuss $s$-polarized incoming light only. In our setup, due to geometrical constraints of the magnet given the laser beam makes only an angle of about $20^{\circ}$ with the normal of the sample surface. This is significantly below the Brewster angle $\theta_{\mathrm{BR}} \approx \arctan \left(n_{2} / n_{1}\right)=63^{\circ}$ when using $\operatorname{Re}\left(n_{2}\right)=2$ for Co metal and $n_{1}=1$ for air. At the latter, the reflection of $p$-polarized light is minimized while the longitudinal Kerr rotation of $s$-polarized light increases linearly with increasing angle of incidence up to $\approx \theta_{\mathrm{BR}}{ }^{34,35}$ The laser beam then passes through a Glan-Thompson polarizer (Edmund Optics) with an extinction coefficient of $10^{-5}$ which produces high degree of polarization. A lens of focal length $f=350 \mathrm{~mm}$ and diameter of $D=25 \mathrm{~mm}$ is used to focus the light beam onto the sample surface. The reflected beam is periodically modulated between left and right circularly polarized light by the photoelastic modulator (PEM-90, Hinds Instruments). Modulation takes place with a frequency of $50 \mathrm{kHz}$ and phase amplitudes of $\varphi_{0}=108^{\circ}$ and $\varphi_{0}=175^{\circ}$ which maximize ${ }^{36}$ the Bessel functions

$$
J_{1}(\varphi)=\sum_{m=0}^{\infty} \frac{(-1)^{m}}{m !(m+1) !}\left(\frac{\varphi}{2}\right)^{2 m+1}
$$

and

$$
J_{2}(\varphi)=\sum_{m=0}^{\infty} \frac{(-1)^{m}}{m !(m+2) !}\left(\frac{\varphi}{2}\right)^{2 m+2}
$$

for first and second harmonic measurements, respectively. The modulation signal is used as reference signal for a lock-in amplifier (Stanford Research Systems, SR830 DSP). The beam then transmits through an analyzer and is finally detected by a photosensitive fast responding diode (DET100, Hinds Instruments) providing the input signal to the lock-in amplifier. A lock-in integration time of $\tau=300 \mathrm{~ms}$ has been chosen in agreement with the sweep rate of the magnetic field such that time averaging is optimized while artificial rounding due to cutoff effects is avoided. Note that all subsequently depicted hysteresis loops are single loops without averaging over multiple data sets.

An electromagnet (GMW 3470) powered by a bipolar power supply (Kepco, BOP 36-12M) generates magnetic fields calibrated by a Hall sensor (model 5080, Sypris Instruments). The sample was mounted on a cryostat (Janis Research, CCS-350H) specimen holder, where the temperature can be varied between 10 and $475 \mathrm{~K}$. The versatile designs of the electromagnet and cryostat allow studying a wide variety of magnetic properties in longitudinal, polar, and transverse geometries. Moreover, Faraday effect and magnetotransport measurements can also be realized. The magnetic field control, intensity measurements, and subsequent data collection were coordinated with self-written LABVIEW-7 (National Instruments) programs. The magnet power supply and the lock-in were controlled with the computer via a General Purpose Interface Bus card.

\section{MATRIX ANALYSIS}

According to the quantum mechanical treatment of MOKE ${ }^{37}$ the Kerr rotation $\theta_{K}$ and the Kerr ellipticity $\varepsilon_{K}$ are in good approximation proportional to the magnetization of the sample. The first and second harmonics of the reflected light intensity are related to the off-diagonal elements $r_{s p / p s}$ of the sample's dielectric tensor and determine $\theta_{K}$ and $\varepsilon_{K}$.

In the following, we describe the principle of MOKE with polarization modulation technique in terms of the Jones matrix method. ${ }^{38}$ Each optical component in Fig. 1 can be expressed by a Jones matrix. All angles are relative to the plane of incidence unless otherwise noted. The matrices of the polarizer $(\mathrm{P})$ and analyzer (A) with major transmission axes oriented at angles $\beta$ and $\alpha$, respectively, with the plane of incidence are 


$$
\underline{\underline{P}}=\left[\begin{array}{cc}
\cos ^{2} \beta & \sin \beta \cos \beta \\
\sin \beta \cos \beta & \sin ^{2} \beta
\end{array}\right]
$$

and

$$
\underline{\underline{A}}=\left[\begin{array}{cc}
\cos ^{2} \alpha & \sin \alpha \cos \alpha \\
\sin \alpha \cos \alpha & \sin ^{2} \alpha
\end{array}\right] .
$$

The matrix describing the magnetic sample is expressed as

$$
\underline{\underline{S}}=\left[\begin{array}{cc}
\tilde{r}_{p} & \tilde{r}_{p s} \\
\tilde{r}_{s p} & \widetilde{r}_{s}
\end{array}\right],
$$

where the diagonal terms, $\widetilde{r}_{p}=r_{p} e^{i \delta_{p}}$ and $\widetilde{r}_{s}=r_{s} e^{i \delta_{s}}$, are independent of magnetization and are identified as usual Fresnel reflection coefficients. The off-diagonal cross terms account for the magneto-optic Kerr effect and are symmetric, i.e.,

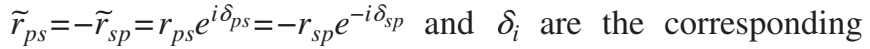
phase angles.

The photoelastic modulator $(\mathrm{O})$ with its axes oriented at $0^{\circ}$, and $90^{\circ}$ is represented by the matrix

$$
\underline{\underline{O}}=\left[\begin{array}{cc}
e^{i \varphi / 2} & 0 \\
0 & e^{-i \varphi / 2}
\end{array}\right],
$$

where the modulator generates a periodic retardation is $\varphi=\varphi_{0} \sin \omega t$. The subsequent analysis requires a Fourier decomposition of $\cos (\varphi(t))$ and $\sin (\varphi(t))$ which reads $\cos (\varphi)=J_{0}\left(\varphi_{0}\right)+2 \sum_{m=1}^{\infty} J_{2 m}\left(\varphi_{0}\right) \cos (2 m \omega t)$ and $\sin (\varphi)$ $=2 \sum_{m=0}^{\infty} J_{2 m+1}\left(\varphi_{0}\right) \sin [(2 m+1) \omega t]$. Here, $J_{k}\left(\varphi_{0}\right)$ are Bessel functions of argument $\varphi_{0}$ and order $k$.

The electric field vector amplitude of the reflected beam $E^{r}$ at the photodetector can be represented by a vector equation,

$$
\left[\begin{array}{l}
E_{p} \\
E_{s}
\end{array}\right]^{r}=\underset{=}{\operatorname{A}} \underline{\underline{S}} \underline{\underline{P}}=\left[\begin{array}{c}
E_{p} \\
E_{s}
\end{array}\right]^{i}
$$

where $E^{i}$ is the amplitude of incident light. $E_{p}$ and $E_{s}$ are the $E$-vector amplitudes in the direction parallel and perpendicular to the plane of incidence, while $\underset{\underline{A}}{,} \underline{\underline{Q}}, \underline{\underline{S}}$, and $\underline{\underline{P}}$ are the matrices representing $A, O, S$, and $P$, respectively.

The signal intensity measured at the detector is thus given by

$$
I \propto\left|E^{r}\right|^{2} .
$$

The $\mathrm{S} / \mathrm{N}$ ratio is obtained from the ratio of the average signal $\left|I_{\text {avg }}^{\text {sat }}\right|$ obtained in a field range where the magnetization of the reference sample is in its saturation state to the average noise $\Delta I^{\mathrm{sat}}=\left(1 / \Delta H I_{\text {avg }}^{\mathrm{sat}}\right) \int_{H}^{H+\Delta H}\left(I^{\mathrm{sat}}(H)-I_{\text {avg }}^{\mathrm{sat}}\right)^{2} d H$ in a measured loop,

$$
S / N=\frac{\left|I_{\text {avg }}^{\text {sat }}\right|}{\left|\Delta I^{\text {sat }}\right|} .
$$

The primarily noise that can be effectively reduced by the modulation technique originates from fluctuations in the polarization of the light caused by fluctuations in the Fresnel reflection coefficients. Note that time dependent misalignments of the light beam with respect to the optical axis are not included in the Jones matrix analysis. Hence, the modulation technique is not effective in noise reduction of mechanical origin. In addition, the efficiency of the modulation technique depends critically on the quality and stability of the modulator. If the latter fluctuates in phase or amplitude the modulation technique can actually add noise to the detected intensity instead of reducing it.

\section{RESULTS AND DISCUSSION}

In the following we consider various meaningful configurations of our MOKE setup. They are distinguished by the order of placements of the optical components and variation of the polarizer/analyzer orientations with respect to each other and with respect to the retardation axis of the modulator. The laser beam transmits through the optical components in the order given in each configuration. The latter is indicated at the beginning of each configuration subsequently analyzed in detail.

\section{A. Configuration 1.1: $P$ transmission axis at $90^{\circ}, S$, $O$ axes at 0 and $90^{\circ}, A$ transmission axis at $\alpha$}

The electric field vector of the reflected light for this configuration following Eq. (5) reads

$$
\begin{aligned}
{\left[\begin{array}{c}
E_{p} \\
E_{s}
\end{array}\right]=} & {\left[\begin{array}{cc}
\cos ^{2} \alpha & \sin \alpha \cos \alpha \\
\sin \alpha \cos \alpha & \sin ^{2} \alpha
\end{array}\right]\left[\begin{array}{cc}
e^{i \varphi / 2} & 0 \\
0 & e^{-i \varphi / 2}
\end{array}\right] } \\
& \times\left[\begin{array}{cc}
\widetilde{r}_{p} & \widetilde{r}_{p s} \\
\tilde{r}_{s p} & \widetilde{r}_{s}
\end{array}\right]\left[\begin{array}{ll}
0 & 0 \\
0 & 1
\end{array}\right]\left[\begin{array}{l}
0 \\
1
\end{array}\right] .
\end{aligned}
$$

The intensity is calculated following Eq. (6) and given by

$$
\begin{aligned}
I \propto & r_{s}^{2} \sin ^{2} \alpha+r_{p s}^{2} \cos ^{2} \alpha+2 J_{0}\left(\varphi_{0}\right) r_{s} r_{p s} \\
& \times \cos \left(\delta_{s}-\delta_{p s}\right) \sin \alpha \cos \alpha+4 J_{1}\left(\varphi_{0}\right) \sin \omega t r_{s} r_{p s} \\
& \times \sin \left(\delta_{s}-\delta_{p s}\right) \sin \alpha \cos \alpha \\
& +4 J_{2}\left(\varphi_{0}\right) \cos 2 \omega t r_{s} r_{p s} \cos \left(\delta_{s}-\delta_{p s}\right) \sin \alpha \cos \alpha \\
& + \text { higher order terms. }
\end{aligned}
$$

Now let's analyze the variation of intensity with different $\alpha$ as shown below.

- Case 1, $\alpha=0$ :

$I \propto r_{p s}^{2}$.

- Case 2, $\alpha=90^{\circ}$ :

$I \propto r_{s}^{2}$.

- Case 3, $\alpha=45^{\circ}$ :

$$
\begin{aligned}
I \propto & \frac{1}{2} r_{s}^{2}+\frac{1}{2} r_{p s}^{2}+J_{0}\left(\varphi_{0}\right) r_{s} r_{p s} \cos \left(\delta_{s}-\delta_{p s}\right) \\
& +2 J_{1}\left(\varphi_{0}\right) \sin \omega t r_{s} r_{p s} \sin \left(\delta_{s}-\delta_{p s}\right) \\
& +2 J_{2}\left(\varphi_{0}\right) \cos 2 \omega t r_{s} r_{p s} \cos \left(\delta_{s}-\delta_{p s}\right) \\
& + \text { higher order terms. }
\end{aligned}
$$

- Case 4, $\alpha=135^{\circ}$ :

$$
\begin{aligned}
I \propto & \frac{1}{2} r_{s}^{2}+\frac{1}{2} r_{p s}^{2}-J_{0}\left(\varphi_{0}\right) r_{s} r_{p s} \cos \left(\delta_{s}-\delta_{p s}\right) \\
& -2 J_{1}\left(\varphi_{0}\right) \sin \omega t r_{s} r_{p s} \sin \left(\delta_{s}-\delta_{p s}\right) \\
& -2 J_{2}\left(\varphi_{0}\right) \cos 2 \omega t r_{s} r_{p s} \cos \left(\delta_{s}-\delta_{p s}\right)
\end{aligned}
$$$$
+ \text { higher order terms. }
$$

Typical first and second harmonic Kerr loops measured for 

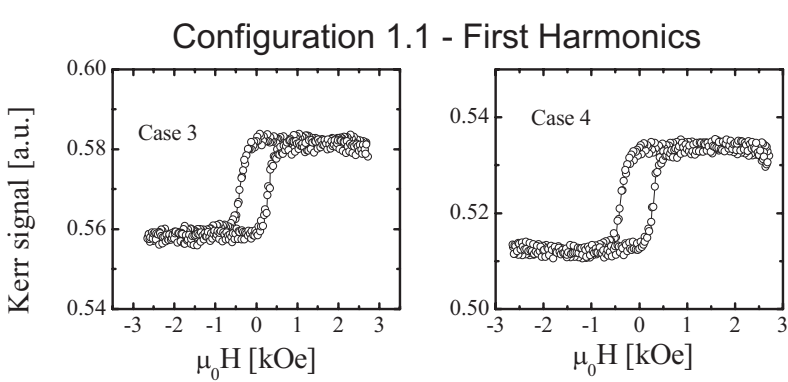

Configuration 1.1 - Second Harmonics
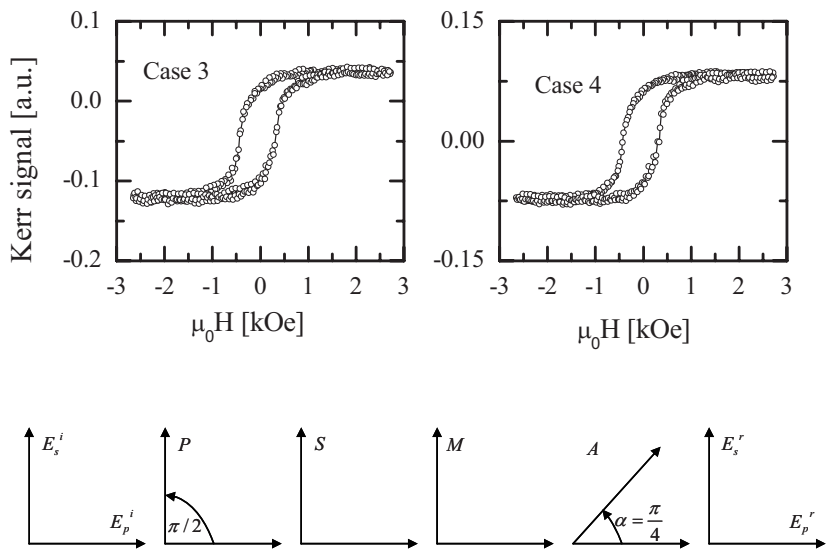

FIG. 2. First and second harmonic MOKE hysteresis loops analyzed by a lock-in amplifier using an integration time constant of $300 \mathrm{~ms}$. Optical setups are in accordance with cases 3 and 4 of configuration 1.1. The bottom sketch shows the change of the initial polarization state of the light into the final polarization when subsequently passing through the optical components of the setup. For definitions of the notation involved see text.

various cases in this configuration are presented in Fig. 2. The fact that no Kerr signal is expected for cases 1 and 2 is corroborated by experimental observation of the measured data. On the other hand, terms containing first and second harmonics for cases 3 and 4 result in signals detected by the lock-in amplifier and, hence, Kerr hysteresis loops. The S/N ratio amounts to 21.7 (first harmonic), 45.9 (second harmonic), and 24.2 (first harmonic), 51.0 (second harmonic) for cases 3 and 4, respectively. The subsequent analysis of various configurations reveals that configuration 1.1, cases 3 and 4 are the optimized setup for longitudinal Kerr measurements of the first as well as the second harmonic.

\section{B. Configuration 1.2: $P$ axis at $90^{\circ}, O$ axes at 0 and $90^{\circ}, S, A$ axis at $\alpha$}

In this case the intensity at the detector is given by

$$
\begin{aligned}
I \propto & r_{s}^{2} \sin ^{2} \alpha+r_{p s}^{2} \cos ^{2} \alpha+2 r_{s} r_{p s} \\
& \times \cos \left(\delta_{s}-\delta_{p s}\right) \sin \alpha \cos \alpha .
\end{aligned}
$$

Analyze the variation of intensity with different $\alpha$ is shown below.

- Case 1, $\alpha=0$ :

$$
I \propto r_{p s}^{2} .
$$

- Case 2, $\alpha=90^{\circ}$ :

$I \propto r_{s}^{2}$.

- Case 3, $\alpha=45^{\circ}$ :

$$
I \propto \frac{1}{2} r_{s}^{2}+\frac{1}{2} r_{p s}^{2}+r_{s} r_{p s} \cos \left(\delta_{s}-\delta_{p s}\right) .
$$

- Case 4, $\alpha=135^{\circ}$ :

$$
I \propto \frac{1}{2} r_{s}^{2}+\frac{1}{2} r_{p s}^{2}-r_{s} r_{p s} \cos \left(\delta_{s}-\delta_{p s}\right) .
$$

As predicted by the theory, no hysteresis loop was observed experimentally due to absence of any time dependence of the signal.

\section{Configuration $2.1 P$ axis at $45^{\circ}, S, O$ axes at 0 and $90^{\circ}, A$ axis at $\alpha$}

Detailed analyses of the calculated intensity following Eq. (6) in this configuration for various cases of $\alpha\left(0^{\circ}, 45^{\circ}\right.$, $90^{\circ}, 135^{\circ}$ ) reveal that for both harmonics the magnetic information in the off-diagonal elements is masked by the dominating diagonal elements $r_{p}$ and $r_{s}$. The loops recorded in this configuration do not display any hysteresis.

\section{Configuration 2.2: $P$ axis at $45^{\circ}, O$ axes at 0 and $90^{\circ}, S, A$ axis at $\alpha$}

A detailed analysis of the calculated intensity in this configuration shows that although magnetic hysteresis loops can be measured, the intensity of both harmonics is reduced by a factor of 4 compared to configuration 1.1. Hence, this is a typical situation of a configuration that works but is far from an ideal $\mathrm{S} / \mathrm{N}$ ratio.

\section{E. Configuration 3.1: $P$ axis at $90^{\circ}, S, O$ axes at $45^{\circ}$ and $135^{\circ}, A$ axis at $\alpha$}

The calculated intensity in this configuration is given by

$$
\begin{aligned}
I \propto & \frac{1}{2} r_{s}^{2}+\frac{1}{2} r_{p s}^{2}+r_{s} r_{p s} \cos \left(\delta_{s}-\delta_{p s}\right) \sin 2 \alpha \\
& -\frac{1}{2} J_{0}\left(\varphi_{0}\right)\left(r_{s}^{2}-r_{p s}^{2}\right) \cos 2 \alpha-2 J_{1}\left(\varphi_{0}\right) \sin \omega t r_{s} r_{p s} \\
& \times \sin \left(\delta_{s}-\delta_{p s}\right) \cos 2 \alpha-J_{2}\left(\varphi_{0}\right) \cos 2 \omega t\left(r_{s}^{2}-r_{p s}^{2}\right) \cos 2 \alpha \\
& + \text { higher order terms. }
\end{aligned}
$$

- Case 1, $\alpha=0$ :

$$
\begin{aligned}
I \propto & \frac{1}{2} r_{s}^{2}+\frac{1}{2} r_{p s}^{2}-\frac{1}{2} J_{0}\left(\varphi_{0}\right)\left(r_{s}^{2}-r_{p s}^{2}\right) \\
& -2 J_{1}\left(\varphi_{0}\right) \sin \omega t r_{s} r_{p s} \sin \left(\delta_{s}-\delta_{p s}\right)-J_{2}\left(\varphi_{0}\right) \\
& \times \cos 2 \omega t\left(r_{s}^{2}-r_{p s}^{2}\right)+\text { higher order terms } .
\end{aligned}
$$

- Case 2, $\alpha=90^{\circ}$ :

$$
\begin{aligned}
I \propto & \frac{1}{2} r_{s}^{2}+\frac{1}{2} r_{p s}^{2}+\frac{1}{2} J_{0}\left(\varphi_{0}\right)\left(r_{s}^{2}-r_{p s}^{2}\right) \\
& +2 J_{1}\left(\varphi_{0}\right) \sin \omega t r_{s} r_{p s} \sin \left(\delta_{s}-\delta_{p s}\right)+J_{2}\left(\varphi_{0}\right) \\
& \times \cos 2 \omega t\left(r_{s}^{2}-r_{p s}^{2}\right)+\text { higher order terms }
\end{aligned}
$$

- Case 3, $\alpha=45^{\circ}$ :

$$
I \propto \frac{1}{2} r_{s}^{2}+\frac{1}{2} r_{p s}^{2}+r_{s} r_{p s} \cos \left(\delta_{s}-\delta_{p s}\right) .
$$

- Case 4, $\alpha=135^{\circ}$ :

$$
I \propto \frac{1}{2} r_{s}^{2}+\frac{1}{2} r_{p s}^{2}-r_{s} r_{p s} \cos \left(\delta_{s}-\delta_{p s}\right) .
$$

The loops measured in this configuration are displayed in Fig. 3. It is noticed that cases 1 and 2 for the first harmonic give rise to high $\mathrm{S} / \mathrm{N}$ ratio such as 36.6 and 47.2 , respectively 

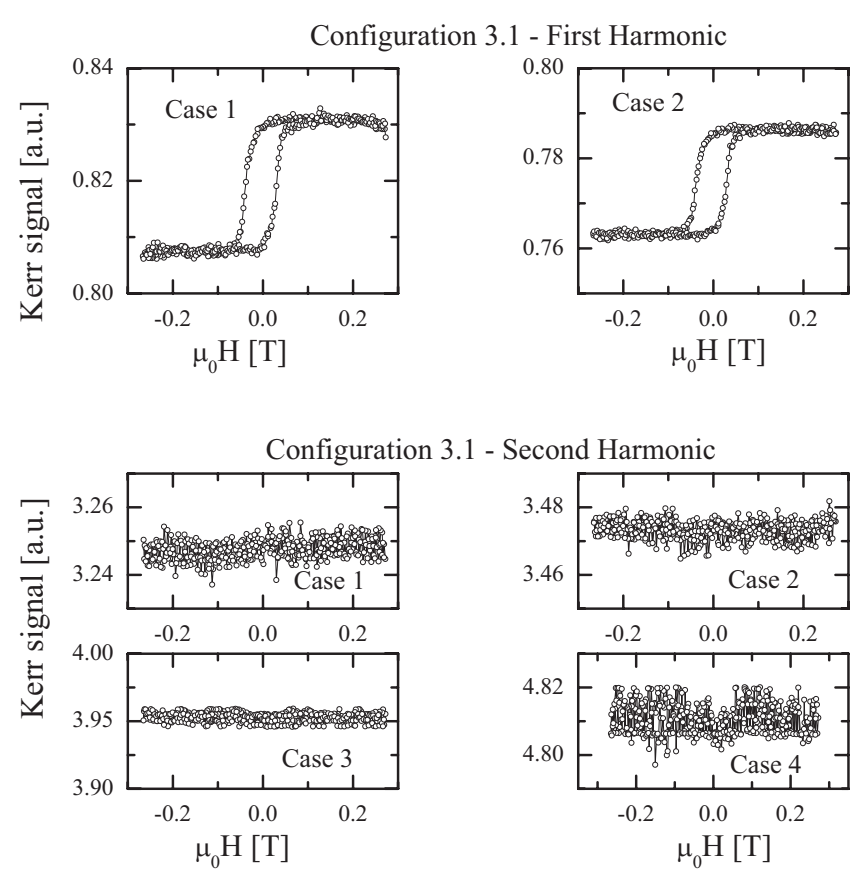

FIG. 3. First and second harmonic MOKE hysteresis loops analyzed by a lock-in amplifier using an integration time constant of $300 \mathrm{~ms}$. Optical setups resemble various cases using configuration 3.1. This setting may be used for measuring the first harmonic, but is non-ideal for measuring the second harmonic loop.

in accordance with the theoretical analysis. In addition the theory predicts that the second harmonic signal in this setting suffers from a large background contribution originating from a diagonal reflection coefficient. This is qualitatively confirmed by the high $\mathrm{S} / \mathrm{N}$ data shown in Fig. 3. This configuration reflects an asymmetric situation and is, hence, not suitable for measurement of both harmonics.

\section{F. Configuration 3.2: $P$ axis at $90^{\circ}, O$ axes at $45^{\circ}$ and $135^{\circ}, S, A$ axis at $\alpha$}

The calculated intensity in this configuration is given by

$$
\begin{aligned}
I= & \frac{1}{2} r_{p}^{2} \cos ^{2} \alpha+\frac{1}{2} r_{s}^{2} \sin ^{2} \alpha+\frac{1}{2} r_{p s}^{2}-r_{p} r_{p s} \\
& \times \cos \left(\delta_{p}-\delta_{p s}\right) \sin \alpha \cos \alpha+r_{s} r_{p s} \cos \left(\delta_{s}-\delta_{p s}\right) \\
& \times \sin \alpha \cos \alpha-\frac{1}{2} J_{0}\left(\varphi_{0}\right)\left[r_{p}^{2} \cos ^{2} \alpha-r_{s}^{2} \sin ^{2} \alpha\right. \\
& -r_{p s}^{2} \cos 2 \alpha-2 r_{p} r_{p s} \cos \left(\delta_{p}-\delta_{p s}\right) \sin \alpha \cos \alpha \\
& \left.+2 r_{s} r_{p s} \cos \left(\delta_{s}-\delta_{p s}\right) \sin \alpha \cos \alpha\right] \\
& -2 J_{1}\left(\varphi_{0}\right) \sin \omega t\left[r_{p} r_{s} \sin \left(\delta_{p}-\delta_{s}\right) \sin \alpha \cos \alpha\right. \\
& \left.+r_{p} r_{p s} \sin \left(\delta_{p}-\delta_{p s}\right) \cos ^{2} \alpha+r_{s} r_{p s} \sin \left(\delta_{s}-\delta_{p s}\right) \sin ^{2} \alpha\right] \\
& -J_{2}\left(\varphi_{0}\right) \cos 2 \omega t\left[r_{p}^{2} \cos ^{2} \alpha-r_{s}^{2} \sin ^{2} \alpha-r_{p s}^{2} \cos 2 \alpha\right. \\
& -2 r_{p} r_{p s} \cos \left(\delta_{p}-\delta_{p s}\right) \sin \alpha \cos \alpha+2 r_{s} r_{p s} \\
& \left.\times \cos \left(\delta_{s}-\delta_{p s}\right) \sin \alpha \cos \alpha\right]+ \text { higher order terms. }
\end{aligned}
$$

- Case 1, $\alpha=0$ :

$$
\begin{aligned}
I \propto & \frac{1}{2} r_{p}^{2}+\frac{1}{2} r_{p s}^{2}-\frac{1}{2} J_{0}\left(\varphi_{0}\right)\left(r_{p}^{2}-r_{p s}^{2}\right) \\
& -2 J_{1}\left(\varphi_{0}\right) \sin \omega t r_{p} r_{p s} \sin \left(\delta_{p}-\delta_{p s}\right)-J_{2}\left(\varphi_{0}\right) \\
& \times \cos 2 \omega t\left(r_{p}^{2}-r_{p s}^{2}\right)+\text { higher order terms. }
\end{aligned}
$$

- Case 2, $\alpha=90^{\circ}$ :

$$
\begin{aligned}
I \propto & \frac{1}{2} r_{s}^{2}+\frac{1}{2} r_{p s}^{2}+\frac{1}{2} J_{0}\left(\varphi_{0}\right)\left(r_{s}^{2}-r_{p s}^{2}\right) \\
& -2 J_{1}\left(\varphi_{0}\right) \sin \omega t r_{s} r_{p s} \sin \left(\delta_{s}-\delta_{p s}\right)+J_{2}\left(\varphi_{0}\right)
\end{aligned}
$$

$\times \cos 2 \omega t\left(r_{s}^{2}-r_{p s}^{2}\right)+$ higher order terms.

- Case 3, $\alpha=45^{\circ}$ :

$$
\begin{aligned}
I \propto & \frac{1}{4} r_{p}^{2}+\frac{1}{4} r_{s}^{2}+\frac{1}{2} r_{p s}^{2}-\frac{1}{2} r_{p} r_{p s} \cos \left(\delta_{p}-\delta_{p s}\right)+\frac{1}{2} r_{s} r_{p s} \\
& \times \cos \left(\delta_{s}-\delta_{p s}\right)-\frac{1}{4} J_{0}\left(\varphi_{0}\right)\left[r_{p}^{2}-r_{s}^{2}-2 r_{p} r_{p s} \cos \left(\delta_{p}-\delta_{p s}\right)\right. \\
& \left.+2 r_{s} r_{p s} \cos \left(\delta_{s}-\delta_{p s}\right)\right]-J_{1}\left(\varphi_{0}\right) \sin \omega t\left[r_{p} r_{s} \sin \left(\delta_{p}-\delta_{s}\right)\right. \\
& \left.+r_{p} r_{p s} \sin \left(\delta_{p}-\delta_{p s}\right)+r_{s} r_{p s} \sin \left(\delta_{s}-\delta_{p s}\right)\right] \\
& -\frac{1}{2} J_{2}\left(\varphi_{0}\right) \cos 2 \omega t\left[r_{p}^{2}-r_{s}^{2}-2 r_{p} r_{p s} \cos \left(\delta_{p}-\delta_{p s}\right)\right. \\
& \left.+2 r_{s} r_{p s} \cos \left(\delta_{s}-\delta_{p s}\right)\right]+ \text { higher order terms. }
\end{aligned}
$$

- Case 4, $\alpha=135^{\circ}$ :

$$
\begin{aligned}
I \propto & \frac{1}{4} r_{p}^{2}+\frac{1}{4} r_{s}^{2}+\frac{1}{2} r_{p s}^{2}+\frac{1}{2} r_{p} r_{p s} \cos \left(\delta_{p}-\delta_{p s}\right)-\frac{1}{2} r_{s} r_{p s} \\
& \times \cos \left(\delta_{s}-\delta_{p s}\right)-\frac{1}{4} J_{0}\left(\varphi_{0}\right)\left[r_{p}^{2}-r_{s}^{2}+2 r_{p} r_{p s} \cos \left(\delta_{p}-\delta_{p s}\right)\right. \\
& \left.-2 r_{s} r_{p s} \cos \left(\delta_{s}-\delta_{p s}\right)\right]+J_{1}\left(\varphi_{0}\right) \sin \omega t\left[r_{p} r_{s} \sin \left(\delta_{p}-\delta_{s}\right)\right. \\
& \left.-r_{p} r_{p s} \sin \left(\delta_{p}-\delta_{p s}\right)-r_{s} r_{p s} \sin \left(\delta_{s}-\delta_{p s}\right)\right] \\
& -\frac{1}{2} J_{2}\left(\varphi_{0}\right) \cos 2 \omega t\left[r_{p}^{2}-r_{s}^{2}+2 r_{p} r_{p s} \cos \left(\delta_{p}-\delta_{p s}\right)\right. \\
& \left.-2 r_{s} r_{p s} \cos \left(\delta_{s}-\delta_{p s}\right)\right]+ \text { higher order terms. }
\end{aligned}
$$

This configuration shows the same asymmetry between the first and second harmonic as configuration 3.1 and is, hence, not ideal.

\section{G. Configuration 4.1: $P$ axis at $45^{\circ}, S, O$ axes at $45^{\circ}$ and $135^{\circ}, A$ axis at $\alpha$}

This configuration for various cases of $\alpha\left(0^{\circ}, 45^{\circ}, 90^{\circ}\right.$, $135^{\circ}$ ) combines the disadvantages of reduced harmonic signals and the masking of the magnetic information by the diagonal elements.

\section{H. Configuration 4.2: $P$ axis at $45^{\circ}, O$ axes at $45^{\circ}$ and $135^{\circ}, S, A$ axis at $\alpha$}

The theoretical calculation of intensity suggests the absence of any signal in good agreement with our experimental findings.

Table I shows the experimental $\mathrm{S} / \mathrm{N}$ ratios obtained for all configurations discussed above.

\section{CONCLUSIONS}

We compared various longitudinal Kerr setups based on polarization modulation methodology using Jones matrix formalism. The predicted signal intensities of the first and second harmonics have been tested with the help of experimental configurations and signal to noise analysis of magnetic hysteresis data. A molecular beam epitaxially grown 
TABLE I. The calculated $\mathrm{S} / \mathrm{N}$ ratio values for meaningful configurations.

\begin{tabular}{lccc}
\hline \hline \multicolumn{2}{c}{ Configurations } & First harmonic & Second harmonic \\
\hline Config. & Case 3 & 21.7 & 45.9 \\
1.1 & Case 4 & 24.2 & 51.0 \\
Config. & Case 1 & 6.7 & 9.5 \\
2.2. & Case 2 & 1.2 & 26.9 \\
Config. & Case 1 & 36.6 & N/A \\
3.1. & Case 2 & 47.2 & N/A \\
Config. & Case 1 & 51.2 & N/A \\
3.2 & Case 2 & 37.3 & N/A \\
\hline \hline
\end{tabular}

$\mathrm{CoO} / \mathrm{Co}$ reference sample has been used throughout all experiments performed at room temperature where exchange bias is absent but appreciable coercivity enhancement is still present increasing the magnetic field range of physical interest. Various configurations give rise to Kerr signals. Some of them have either optimized first or second harmonic signals. Others show reduced signal to noise ratios due to large fieldindependent contributions originating from the diagonal elements of the dielectric tensor. The optimized setup (configuration 1.1) stands out by maximizing the signal of the first and the second harmonics and is free from nonmagnetic background contributions.

\section{ACKNOWLEDGMENTS}

This research work is supported by NSF through Career DMR-0547887, the Nebraska Research Initiative (NRI), and by the MRSEC Program of the NSF (DMR-0213808).

${ }^{1}$ J. Kerr, Philos. Mag. 3, 321 (1877).

${ }^{2}$ S. D. Bader, J. Magn. Magn. Mater. 100, 440 (1991).

${ }^{3}$ Ultrathin Magnetic Structures, edited by B. Heinrich and J. A. C. Bland (Springer, Berlin, 1994).

${ }^{4}$ J. L. Erskine and E. A. Stern, Phys. Rev. Lett. 30, 1329 (1973).

${ }^{5}$ P. Grünberg, R. Schreiber, Y. Pang, U. Walz, M. B. Brodsky, and H. Sowers, J. Appl. Phys. 61, 3750 (1987).

${ }^{6}$ M. T. Johnson, R. Coehoorn, J. J. de Vries, N. W. McGee, J. aan de Stegge, and P. J. H. Bloemen, Phys. Rev. Lett. 69, 969 (1992).
${ }^{7}$ E. E. Fullerton, M. J. Conover, J. E. Mattson, C. H. Sowers, and S. D. Bader, Phys. Rev. B 48, 15755 (1993).

${ }^{8}$ C. Liu, E. R. Moog, and S. D. Bader, Phys. Rev. Lett. 60, 2422 (1988).

${ }^{9}$ J. Kohlhepp, H. J. Elmers, S. Cordes, and U. Gradmann, Phys. Rev. B 45, 12287 (1992).

${ }^{10}$ Z. Q. Qiu, J. Pearson, and S. D. Bader, Phys. Rev. Lett. 70, 1006 (1993).

${ }^{11}$ A. Berger and H. Hopster, Phys. Rev. Lett. 76, 519 (1996).

${ }^{12}$ H. P. Oepen, M. Speckmann, Y. T. Millev, and J. Kirschner, Phys. Rev. B 55, 2752 (1997)

${ }^{13}$ M. A. Torija, J. P. Pierce, and J. Shen, Phys. Rev. B 63, 092404 (2001).

${ }^{14}$ J. Chen and J. Erskine, Phys. Rev. Lett. 68, 1212 (1992).

${ }^{15}$ Z. Q. Qiu and S. D. Bader, Rev. Sci. Instrum. 71, 1243 (2000), and references therein.

${ }^{16}$ A. Berger, and M. R. Pufall, Appl. Phys. Lett. 71, 965 (1997).

${ }^{17}$ V. Usov, S. Murphy, L. Seravalli, and I. V. Shvets, Rev. Sci. Instrum. 76, 046102 (2005)

${ }^{18}$ J. R. Hampton, J.-L. Martínez-Albertos, and H. Abruna, Rev. Sci. Instrum. 73, 3018 (2002)

${ }^{19}$ K. M. Poduska and S. Morin, Rev. Sci. Instrum. 74, 4723 (2003).

${ }^{20}$ D. A. Allwood, G. Xiong, M. D. Cooke, and R. P. Cowburn, J. Phys. D 36, 2175 (2003).

${ }^{21}$ T. Ishibashi, Z. Kuang, S. Yufune, T. Kawata, M. Oda, T. Tani, Y. Iimura, K. Sato, Y. Konishi, K. Akahane, X. Zhao, and T. Hasegawa, J. Appl. Phys. 100, 093903 (2006).

${ }^{22}$ J. Hamrle, J. Ferré, J. P. Jamet, V. Repain, G. Baudot, and S. Rousset, Phys. Rev. B 67, 155411 (2003).

${ }^{23}$ A. V. Petukhov, A. Kirilyuk, and Th. Rasing, Phys. Rev. B 59, 4211 (1999).

${ }^{24}$ W. Kleemann, Rev. Sci. Instrum. 78, 120901 (2007).

${ }^{25}$ W. Westphalen, M.-S. Lee, A. Remhof, and H. Zabel, Rev. Sci. Instrum. 78, 121301 (2007).

${ }^{26}$ K. Sato, Jpn. J. Appl. Phys. 20, 2403 (1981).

${ }^{27}$ S. N. Jesperson and S. E. Schnatterly, Rev. Sci. Instrum. 40, 761 (1969).

${ }^{28}$ P. Q. J. Nederpel and J. W. D. Martens, Rev. Sci. Instrum. 56, 687 (1985).

${ }^{29}$ W. S. Kim, M. Aderholz, and W. Kleemann, Meas. Sci. Technol. 4, 1275 (1993).

${ }^{30}$ S. Maat, L. Shen, C. Hou, H. Fujiwara, and G. J. Mankey, J. Appl. Phys. 85, 1658 (1999).

${ }^{31}$ M. Farle, W. A. Lewis, and K. Baberschke, Appl. Phys. Lett. 62, 2728 (1993).

${ }^{32}$ S. Polisetty, S. Sahoo, and Ch. Binek, Phys. Rev. B 76, 184423 (2007).

${ }^{33}$ S.-K. Kim, J.-W. Lee, S.-C. Shin, and K. Y. Kim, J. Appl. Phys. 91, 3099 (2002).

${ }^{34}$ M. N. Deeter and D. Sarid, IEEE Trans. Magn. 24, 2470 (1988).

${ }^{35}$ S. Tanaka, Jpn. J. Appl. Phys. 2, 548 (1963).

${ }^{36}$ G. Arfken, Mathematical Methods for Physicists, 3rd ed. (Academic, Orlando, FL, 1985).

${ }^{37}$ P. N. Argyres, Phys. Rev. 97, 334 (1955).

${ }^{38}$ R. M. A. Azzam and N. M. Bashara, Ellipsometry and Polarized Light (North-Holand, Amsterdam, 1979). 